\title{
Immunophenotyping in Chronic Myeloid Leukemia Blast Crisis: Looking beyond Morphology
}

\author{
${ }^{1}$ Vikram Narang, ${ }^{2}$ Man US Sachdeva, ${ }^{3}$ Parveen Bose, ${ }^{4}$ Neelam Varma, ${ }^{5}$ Pankaj Malhotra, ${ }^{6}$ Subhash Varma
}

\section{ABSTRACT}

Introduction: Patients of chronic myeloid leukemia $(\mathrm{CML})$ eventually transform to blast crisis (BC) after a variable length of chronic phase (CP). With improvement in management protocols, many patients of CML-BC are expected to show remission after initial chemotherapy and a better survival. Hence, it becomes imperative to correctly classify the nature of $\mathrm{BC}$ for further course of management.

Materials and methods: This study retrospectively analyzed cases of CML-BC, over a period of 5 years, with comprehensive immunophenotyping done on flow cytometry.

Results: Fifteen cases of CML-BC with male-to-female ratio of 3:1 and mean age of 40.2 years were included in the study. Flow cytometry revealed 14 cases of myeloid and one case of lymphoid BC. One case each of megakaryocytic and myelomonocytic lineage were seen among the 14 cases of myeloid BC. Aberrant marker expression was a common occurrence. Myeloperoxidase cytochemical stain had shown positivity only in 3 of the 14 cases of myeloid BC.

Conclusion: Immunophenotyping is necessary for assigning specific lineage to blasts in patients of CML-BC and thus providing clinically useful information regarding the treatment protocols and prognosis of the patient.

Keywords: Blast crisis, Chronic myeloid leukemia, Immunophenotyping.

How to cite this article: Narang V, Sachdeva MUS, Bose P, Varma N, Malhotra P, Varma S. Immunophenotyping in Chronic Myeloid Leukemia Blast Crisis: Looking beyond Morphology. J Postgrad Med Edu Res 2016;50(4):181-184

Source of support: Nil

Conflict of interest: None

\footnotetext{
${ }^{1}$ Assistant Professor, ${ }^{2}$ Associate Professor, ${ }^{3}$ Senior Laboratory Technician, ${ }^{4-6}$ Professor

${ }^{1}$ Department of Pathology, Dayanand Medical College and Hospital, Ludhiana, Punjab, India

${ }^{2-4}$ Department of Hematology, Postgraduate Institute of Medical Education and Research, Chandigarh, India

${ }^{5,6}$ Department of Internal Medicine, Postgraduate Institute of Medical Education and Research, Chandigarh, India

Corresponding Author: Man US Sachdeva, Associate Professor, Department of Hematology, Postgraduate Institute of Medical Education and Research, Chandigarh, India, Phone: +917087008132, e-mail: drmanupdeshpgi@yahoo.co.in
}

\section{INTRODUCTION}

Chronic myeloid leukemia (CML) is a myeloproliferative disorder that results from the clonal expansion of a pluripotent stem cell. It is characterized by the presence of balanced translocation, i.e., $\mathrm{t}(9 ; 22)$, leading to formation of a fusion transcript - "BCR-ABL". The identification of this transcript and providing a correct diagnosis becomes extremely important due to the efficacy of "signal transduction inhibitors," like Imatinib, to target the fusion protein and produce remarkable improvement in the course of the disease. The natural clinical course of CML is generally triphasic; initial chronic phase $(\mathrm{CP})$, followed by accelerated phase and subsequent blast crisis (BC), which is an inevitably terminal event. The BC of CML is reported to have cell line heterogeneity, since the blasts can express myeloid, lymphoid, monocytic, megakaryocytic, and rarely erythroid phenotype. Immunophenotyping per se has a limited role in diagnosis of CML but is being used frequently in CML-BC cases. The diverse lineages seen in the $\mathrm{BC}$ have only been noted with the advent and routine use of multicolor flow cytometry. A comprehensive panel of antibodies used in the primary screening makes the lineage determination easy and allows assessment of aberrant expression of antigens. ${ }^{1,2}$ This study was planned to evaluate immunophenotyping results of flow cytometry done for cases of CML-BC at a single tertiary care institute of North India.

\section{MATERIALS AND METHODS}

This study was conducted in the Department of Hematology of Postgraduate Institute of Medical Education and Research, Chandigarh, India. A retrospective analysis over a time period of 5 years (January 2007-April 2012) was carried out to select all cases of CML-BC. Here, BC was defined as the presence of at least $20 \%$ blast in the peripheral blood or bone marrow mononuclear cells. A comprehensive analysis of data retrieved from the archives of the department was carried out. Cases diagnosed as CML-BC with no or incomplete flow cytometry data were excluded from the study. A total of 15 cases of CML-BC were selected for further analysis. All of the selected cases had a confirmation of the diagnosis of CML on the basis of conventional cytogenetics showing Philadelphia 
chromosome and/or presence of bcr-abl fusion gene on RT-PCR.

A review of peripheral blood smears, bone marrow aspirate smears, and bone marrow trephine biopsies of all 15 cases was carried out. Cytochemical stains, i.e., myeloperoxidase (MPO) and periodic acid Schiff (PAS) were carefully reviewed to assist the characterization of the blasts cytochemically.

The immunophenotyping performed by flow cytometry in all 15 cases was also reviewed. The EDTAanticoagulated bone marrow aspirate sample was used in 13 cases and peripheral venous blood sample was used in two cases for immunophenotyping. The samples for flow cytometry were processed as per standardized "lyse-stain-wash" technique routinely used for all acute leukemia cases. Briefly, $100 \mu \mathrm{L}$ of peripheral blood/bone marrow aspirate sample was mixed with $2 \mathrm{~mL} \mathrm{RBC} \mathrm{lysing}$ solution and then centrifuged at $1000 \mathrm{rpm}$ for 5 minutes. The supernatant was discarded and the cell button was further washed in phosphate buffer saline (PBS) solution and subsequently suspended in $500 \mu \mathrm{L}$ of PBS. In $100 \mu \mathrm{L}$ of cell suspension, standardized pre-titrated volumes of monoclonal antibody-cocktails (Becton Dickinson, USA) were added and incubated in dark. The antibody panel comprised of CD19, CD10, CD20, cytoplasmic-CD79a, CD3, CD4, CD7, CD8, cytoplasmic-CD3, CD13, CD33, CD117, antiMPO, CD14, CD64, CD11c, CD41a, CD61, CD235a, CD34, HLADR, and terminal deoxynucleotidyl transferase (TdT). These monoclonal antibodies were used in conjugation with four fluorochromes, i.e., FITC, $\mathrm{PE}, \mathrm{APC}$, and PerCP in each tube. CD45PerCP was used as an anchor marker to assist gating of blasts in all the tubes. The samples were acquired on dual laser BD FACS - Canto II and the analysis was done by FACS Diva software. The flow cytometry data was analyzed, and the lineage of the blasts was determined in each case depending upon the expression of lineage specific markers.

\section{RESULTS}

Fifteen cases of CML-BC were comprehensively immunophenotyped by flow cytometry in 5 years and these were selected for the present study. Thirteen were diagnosed as CML-BC at the time of presentation and two were on treatment with Imatinib. The mean age of the patients included was $40.2 \pm 17(16-74)$ years. There were 11 males and 3 females (M:F: 3:1) (Table 1). Blast percentage in bone marrow aspirate smears ranged from 27 to $60 \%$, with mean of $42.6 \pm 13.4 \%$. Cytochemical staining revealed positivity of MPO in three cases indicating myeloid lineage. Lineage assignment was not possible for the rest of the 12 cases on the basis of morphology and the cytochemistry performed. Fourteen cases were diagnosed as myeloid blast crises, with twelve of these cases expressing markers of granulocytic differentiation, one each with myelomonocytic and megakaryocytic differentiation. Only one case was diagnosed as B-lineage BC. CD33 was the commonest myeloid marker noted in cases diagnosed as myeloid blast crises. This was followed by CD13 expression. None of the cases showed presence of more than one lineage (according to WHO 2008 criteria of lineage assignment) although aberrant antigen expression was commonly noted. Most common aberrancy was expression of CD7 in five cases of myeloid blast crises. Minor population of blasts expressed monocytic and megakaryocytic markers in two separate cases, besides one case each diagnosed as myelomonocytic leukemia and megakaryoblastic leukemia. In addition, two cases of myeloid blast crises expressed a minor population of blasts with erythroid lineage marker (CD235a) (Graph 1).

Table 1: Demography, morphological assessment and lineage assignment by immunophenotyping in 15 cases of CML BC

\begin{tabular}{cllllll}
\hline Sl. no. & Age & Sex & $\begin{array}{l}\text { TLC } \\
\left(\times 10^{9} / L\right)\end{array}$ & $\begin{array}{l}\text { Blast count in bone marrow } \\
\text { aspirate smears }(\%)\end{array}$ & $\begin{array}{l}\text { Myeloperoxidase on } \\
\text { cytochemistry }\end{array}$ & $\begin{array}{l}\text { Lineage assignment on } \\
\text { immunophenotyping }\end{array}$ \\
\hline 1 & 32 & M & 19 & 35 & Positive & Myeloid \\
2 & 35 & F & 15.3 & 32 & Positive & Myeloid \\
3 & 58 & M & 25.7 & 60 & Negative & M lineage \\
4 & 43 & M & 20 & 60 & Megakaryocytic \\
5 & 26 & F & 50.1 & 36 & Positive & Myeloid \\
6 & 27 & M & 56 & 60 & Negative & Myelomonocytic \\
7 & 50 & M & 40.4 & 30 & Negative & Myeloid \\
8 & 42 & M & 51.5 & 29 & Negative & Myeloid \\
9 & 58 & F & 33.2 & 30 & Negative & Myeloid \\
10 & 40 & M & 39.6 & 27 & Negative & Myeloid \\
11 & 24 & M & 76 & 60 & Negative & Myeloid \\
12 & 74 & M & 42 & 38 & Negative & Myeloid \\
13 & 36 & M & 33.6 & 40 & Negative & Myeloid \\
14 & 16 & F & 79.9 & 42 & Negative & Myeloid \\
15 & 42 & M & 15 & 60 & &
\end{tabular}




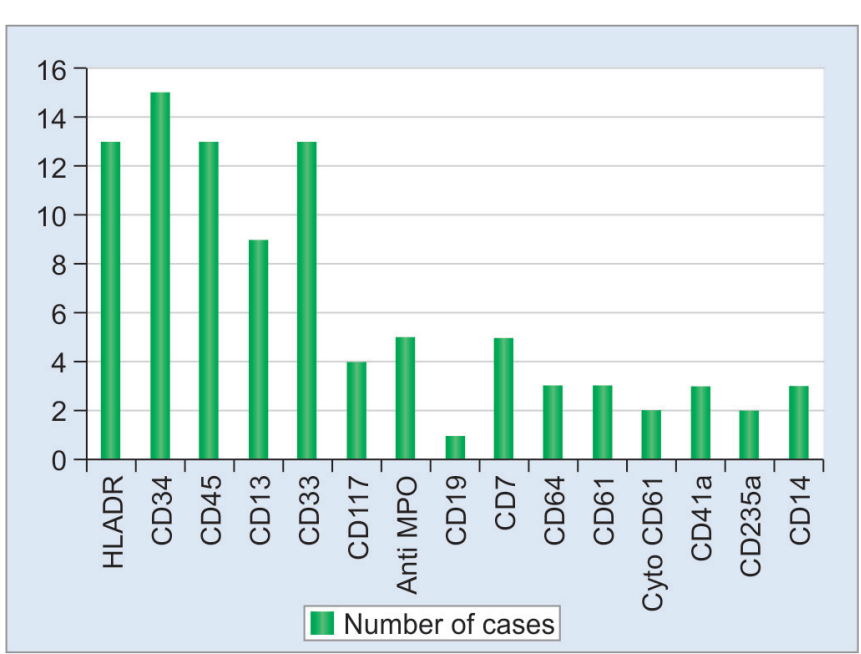

Graph 1: Frequency of occurrence of all the positive markers in the 15 cases of CML blast crises

\section{DISCUSSION}

This retrospective study analyzed 15 cases of CML-BC seen over a period of 5 years and selected on the basis of the availability of comprehensive immunophenotyping on flow cytometry. The median age of patients was 40.9 years and there was preponderance of males $(\mathrm{M}: \mathrm{F}=3: 1)$. The bone marrow smears of these cases were reviewed for assessment of morphology as well as cytochemistry. In all 15 cases of CML-BC, flow cytometry played an important role in determining the nature of blasts and hence, in classifying BC. Fourteen cases were myeloid $\mathrm{BC}$ and only one case was a lymphoid (B-lineage) BC. Only 3 out of 14 cases of myeloid-BC showed MPO positivity on cytochemistry. A low sensitivity of MPO cytochemical staining in cases of CML-BC have been documented earlier, ${ }^{3,4}$ and hence, reliability only on cytochemical MPO is not warranted. Padmanabhan et $\mathrm{al}^{3}$ from the same institute had carried out a similar study on 25 cases of CML-BC. Their patient cohort had a similar mean age as compared to the present study; however, the male-to-female ratio was 1:1.7. They reported low sensitivity of cytochemical stains including MPO, chloroacetate esterase (CAE), Sudan Black B (SBB), and PAS in cases of CML-BC. The authors had done immunophenotyping using indirect immunofluorescence technique on bone marrow aspirate smears and could demonstrate its importance in categorizing the nature of $\mathrm{BC}$.

The present study noted 14 cases of myeloid lineage, and only one case of B-lineage BC. Evolution of CML into $\mathrm{BC}$ is commonly of myeloid type and less frequently of lymphoid type. ${ }^{5}$ We had only one case of lymphoid$\mathrm{BC}$, and the marked preponderance of myeloid $\mathrm{BC}$ in our series might just represent a bias due to small sample size.

There was no case of mixed-lineage BC, defined according to WHO 2008 criteria of lineage assignment; however, majority of cases did show cross lineage antigen expression. CD7 was most common aberrancy $(5 / 14)$ seen in the cases of myeloid BC. In addition to cross antigen expression, heterogeneity of blasts in CML-BC is a wellknown phenomenon. ${ }^{2,3,6,7}$ The malignant event triggering the $\mathrm{BC}$ in CML cases occurs in pleuripotent stem cell, leading to differentiation along different cell lineages, thus explaining the heterogeneity of the blast population.? Saikia et al ${ }^{7}$ in their series of 60 cases of CML-BC, reported five cases showing heterogeneity in the blast population. Griffin et $\mathrm{al}^{2}$ and Bettelheim et $\mathrm{al}^{8}$ have also reported cases with mixed lineage $\mathrm{BC}$ in their respective studies. In contrast, we have not noted a mixed-lineage BC, which is likely because of strict criteria being used for lineage assignment, as compared to studies done prior to introduction of WHO 2008 criteria. However, minor populations of erythroid lineage ( 2 cases), megakaryocytic (1 case), and monocytic lineage (1 case) were noted in separate cases of myeloid BC. Although such cases cannot be classified as mixed-lineage $B C$, immunophenotyping by flow cytometry helps in identifying such minor populations, which might become relevant during course of treatment.

The present study revealed surface expression of CD34 in all our cases of myeloid BC $(n=14)$ and CD7 expression was noted in five cases. CD34 expressing myeloid precursors normally express CD7 transiently and CD34+CD7+ myeloid precursors have been noted at higher frequency in $\mathrm{CP}$ of $\mathrm{CML}$ as compared to normal donors. ${ }^{9}$ Co-expression of CD34 and CD7 suggests that the blasts in cases of CML-BC are arrested at early stage of immature myeloid progenitors. ${ }^{10}$

Paietta and Schwarzmeier ${ }^{11}$ had demonstrated immunophenotypic shifts during the course of therapy in their cohort of CML-BC patients. The phenomenon of alterations in the expression profile of surface markers on blasts after chemotherapy is not uncommon even in acute leukemia cases. However, such immunophenotypic shifts might occur in CML-BC patients on chemotherapy due to the emergence of new clone or previously minor clone, resistant to the therapy. The clonal evolution cannot be picked up by morphology alone and emphasizes the role of immunophenotyping in CML-BC.

Kantarjian et $\mathrm{al}^{12}$ had analyzed a large cohort of 242 patients of CML-BC in the late 1980s. Out of 195 patients who were treated for $\mathrm{BC}$, remission was achieved only in $45(23 \%)$ patients. The overall survival of patients of CML-BC still remains poor. The present study is limited by the unavailability of follow-up of the patients.

Overall, immunophenotyping remains necessary for assigning specific lineage to blasts in patients of CML-BC and thus providing clinically useful information regarding the treatment protocols and prognosis of the patient. Its use is more pertinent since occurrence of mixed lineage blasts in CML-BC is not very rare. 


\section{REFERENCES}

1. Wadhwa J, Szydlo RM, Apperley JF, Chase A, Bua M, Marin D, Olavarria E, Kanfer E, Goldman JM. Factors affecting duration of survival after onset of blastic transformation of chronic myeloid leukemia. Blood 2002 Apr 1;99(7): 2304-2309.

2. Griffin JD, Todd RF 3rd, Ritz J, Nadler LM, Canellos GP, Rosenthal D, Gallivan M, Beveridge RP, Weinstein H, Karp D, et al. Differentiation patterns in the blastic phase of chronic myeloid leukemia. Blood 1983 Jan;61(1):85-91.

3. Padmanabhan V, Varma N, Dash S, Varma S. Role of immunophenotyping in characterisation of blast crisis of chronic myeloid leukemia - a study of 25 cases. Indian J Pathol Microbiol 1996 Jan;39(1):13-17.

4. Favre G, Passweg J, Hoffmann T, Speck B, Gratwohl A, Tichelli A. Immunophenotype of blast crisis in chronic myeloid leukemia. Schweiz Med Wochenschr 1998 Oct 17;128(42):1624-1626.

5. Cervantes F, Villamor N, Esteve J, Montoto S, Rives S, Rozman C, Montserrat E. 'Lymphoid' blast crisis of chronic myeloid leukaemia is associated with distinct clinicohaematological features. Br J Haematol 1998 Jan;100(1):123-128.

6. Tang X, Wu D, Xue Y, Zhu M, Lu D, Ruan C. The morphological, immunophenotypical and cytogenetic characteristics study of blast crisis in chronic myeloid leukemia. Zhonghua Nei Ke Za Zhi 2002 Oct;41(10):685-687.

7. Saikia T, Advani S, Dasgupta A, Ramakrishnan G, Nair C, Gladstone B, Kumar MS, Badrinath Y, Dhond S. Characterisation of blast cells during blastic phase of chronic myeloid leukaemia by immunophenotyping - experience in 60 patients. Leuk Res 1988;12(6):499-506.

8. Bettelheim P, Lutz D, Majdic O, Paietta E, Haas O, Linkesch W, Neumann E, Lechner K, Knapp W. Cell lineage heterogeneity in blast crisis of chronic myeloid leukaemia. Br J Haematol 1985 Mar;59(3):395-409.

9. Kosugi N, Tojo A, Shinzaki H, Nagamura-Inoue T, Asano S. The preferential expression of CD7 and CD34 in myeloid blast crisis in chronic myeloid leukemia. Blood 2000 Mar 15;95(6):2188-2189.

10. Wood BL. Flow cytometric diagnosis of myelodysplasia and myeloproliferative disorders. J Biol Regul Homeost Agents 2004 Apr-Jun;18(2):141-145.

11. Paietta E, Schwarzmeier JD. Phenotypic changes in a case of blast crisis of CML: characterization by TdT, cytochemistry, and cytogenetics. Scand J Haematol 1981 Oct;27(4):241-246.

12. Kantarjian HM, Keating MJ, Talpaz M, Walters RS, Smith TL, Cork A, McCredie KB, Freireich EJ. Chronic myelogenous leukemia in blast crisis. Analysis of 242 patients. Am J Med 1987 Sep;83(3):445-454. 